\title{
Play-Personas: Behaviours and Belief Systems in User-Centred Game Design
}

\author{
Alessandro Canossa ${ }^{1}$ and Anders Drachen ${ }^{2}$ \\ ${ }^{1}$ Denmark Design School, Strandboulevarden 472100 Copenhagen $\varnothing$ \\ IO Interactive, Kalvebod Brygge 4, 1354 Copenhagen K, Denmark \\ aca@dkds.dk, alessandroc@ioi.dk \\ ${ }^{2}$ Center for Computer Games Research, IT University of Copenhagen, Rued Langgaards \\ Vej 7, 2300 Copenhagen S, Denmark \\ anty@itu.dk
}

\begin{abstract}
Game designers attempt to ignite affective, emotional responses from players via engineering game designs to incite definite user experiences. Theories of emotion state that definite emotional responses are individual, and caused by the individual interaction sequence or history. Engendering desired emotions in the audience of traditional audiovisual media is a considerable challenge; however it is potentially even more difficult to achieve the same goal for the audience of interactive entertainment, because a substantial degree of control rests in the hand of the end user rather than the designer. This paper presents a possible solution to the challenge of integrating the user in the design of interactive entertainment such as computer games by employing the "persona" framework introduced by Alan Cooper. This approach is already in use in interaction design. The method can be improved by complementing the traditional narrative description of personas with quantitative, data-oriented models of predicted patterns of user behaviour for a specific computer game Additionally, persona constructs can be applied both as design-oriented metaphors during the development of games, and as analytical lenses to existing games, e.g. for evaluation of patterns of player behaviour.
\end{abstract}

Keywords: Play persona, emotion, game design, user centered design, user experience design.

\section{Introduction: Users and Computer Games}

End users of electronic interactive entertainment such as computer games are accustomed to expect a high degree of customization from the products during actual use. They expect sensitivity, awareness and responsiveness, with the standard being set by Web 2.0 [23] compliant applications and services such as Wikipedia, Google Maps and Facebook. The essence of the innovations posed by these applications is how they place the user at the center of the experience, and how they make the user feel: users think they matter, that they can make a difference and it gives them a way to express themselves. For example Nicole Lazzaro recognized the importance of involving players, at least conceptually, when designing games by stating: "A game's value proposition is how it makes its players think and feel. Players don't buy games, they 
buy experiences" [11]. A failure to incorporate means for mass customization [16] could risk alienating the large majority of people that are becoming more and more acquainted with the practice of expressing themselves at almost every occasion. Furthermore, non-trivial choices and ability to express oneself are seen as determining factors for critical acclaim and are required for adding player choice and broad appeal to computer games [21].

Implementing these innovations in computer games requires both substantial technological advancements and a philosophy of game design that directly incorporates the user needs from the early stages of the design and development process [13, 15, $10,5,22]$. Technological advancements can include platforms for user generated content, adaptive AI, eventually even biometric feedback [17, 18, 14, 12]. We are only starting to scratch the surface of the many possibilities unleashed by user-aware technology. For example, Left4Dead's [1a] AI Director is a dynamic system that monitors the players' performance and orchestrates accordingly the distribution of enemies, items, visual effects, dynamic music and dialogues between characters. Little Big Planet's [2a] magic relies almost completely on the global exchange of its users' creativity, similarly a big portion of the appeal of games such as Spore [3a] or SingStar [4a] seems to be the possibility of sharing user generated content. All these technology-based innovations require considerable investment of resources. On the other hand, a paradigm shift in game design philosophy appears to be emerging, which can bring about a strong focus on the user and create a set of games that attempt to empower players, all of this requiring minimal technical efforts. Such a game design philosophy mirrors what has already happened in the field of Human Computer Interaction: the introduction of User-centered and Experience design [15, 10]. In order to accelerate this paradigm shift in the field of game design this paper advocates first the adaptation and then the adoption of tools and practices already in use in $\mathrm{HCI}$ for modeling users, such as the persona framework $[2,3]$.

\section{Designing User Experience in Games}

According to Frijda [7], emotions are complex dynamic processes and arise as states of action-readiness, including affect and arousal, when we interact with people and things in the world. Past experiences are evaluated and remembered and influence the appraisal of future occurrences to inform ensuing behaviour.

Damasio defines emotional processes as sets of rational, bodily, and behavioural responses to the perception (or memory) of an experience [4].

It is possible to see how, as Sanders correctly points out [19], all efforts aimed at engendering defined experiences and emotions are doomed to failure since experiences and emotional responses alike are too individual, subjective and rooted in people's past to be able to scientifically aim at re-producing them.

It is necessary then to utilize a design philosophy that takes into account players and allows them a certain leeway for expressing themselves, but without assuming universal emotional responses to experiences. Such a frame of mind should be accountable for different player's motivations, goals, behaviours and belief-systems. In the domain of user centred design, such a mental tool exists already. Alan Cooper developed a method called Goal-Directed design [2]. This method makes use of 
personas - "archetypes that represent distinct groupings of behaviours, attitudes, aptitudes, goals, and motivations" [3] - to help developers understand the end user and to foresee its way of interacting with the product.

\subsection{Origin and History of Personas}

Persona is a Latin word and it indicates the mask that actors put on before becoming their characters, it's a socially agreed convention used to represent certain types. Currently it refers mostly to "social masks" or roles that all humans have to play on the stage of life [8]. Goffman uses the term "fronts" to address the different masks that we have to wear according to the different contexts we are presented with. We must act differently in different settings, as the world is a stage. It is in this sense that Jung listed it as one of the archetypes populating the human unconscious.

Personas or fictional identity-constructs have been recognized as fundamental in many creative practices. In literary theory, Iser [9] introduced the term "implied reader" to address the certain "reader that a given literary work requires". Within the frame and the context imposed by the text, this implied reader makes assumptions, has expectations, defines meanings that are left unstated and adds details to characters and settings through a "wandering viewpoint". For example, by Joyce's own admission, Finnegan's Wake should be read by "that ideal reader suffering from an ideal insomnia". Eco expanded on the concept introducing the "model reader" [6] as "the author's foreshadowing of a reader competent enough to provide the best interpretation of a text". The author tries to prefigure a model reader by imagining what could be the actualization of the text. The author, consciously or not, is concerned with how the text / type becomes interpretation / token.

In social sciences, Max Weber introduced the concept of Idealtyp as: "Formed by one-sided accentuation of one or more points of view and by the synthesis of a great many diffuse, discrete, more or less present and occasionally absent concrete individual phenomena, which are arranged according to those one-sidedly emphasized viewpoints into a unified analytical construct" [20]. The ideal type is a pure mental construct used to assess the behaviour of social groups. It is totally theoretic, almost fictitious and generally not empirically found anywhere in reality, it is not backed by statistical data nor a model personality profile, it's more used as some sort of unit of measure, standards much like "meter", "second" or "kilogram" not really found in nature, but useful to measure it.

In similar ways game designers could benefit greatly by making assumptions on the nature of players using personas to map the extreme boundaries of the field of possibilities afforded by their game.

\subsection{Traditional Persona Modeling}

Alan Cooper's [3] goal directed design process starts with the research phase, in which behaviours patterns and modes of use of products are identified. These patterns suggest goals and motivations and in turn these inform the creation of personas. Personas are detailed, composite user archetypes and they serve as main characters in narrative, scenario-based descriptions that iteratively inform the design of a product, so that features emerge directly from the goals. 
Typically a persona is a description of behaviour patterns, goals, skills, attitudes, and environment, with a few fictional personal details to make it a realistic character. For each product there should be set of 3 to 12 personas, it's not necessary to design for all of them, but an extensive cast helps articulate the user population, the primary focus for the design will be a limited subset of maximum three personas. Persona description should be precise including as many details as possible, but not necessarily accurate, it does not need to represent a real person. Name, physical appearance, education, and idiosyncrasies should be included.

The main benefits of personas for product development purposes are:

A) It is easier to relate to a personal human face and name instead of abstract customer data.

B) It is possible to infer user needs not openly stated by drawing on personal peopleexperience.

C) Personas provide a shared, fast and effective language for communication between engineers and designers.

D) Personas states what a user needs and wants so that no stakeholder can reshape the user to their convenience.

E) Personas avoids self-referential designs, where designers might unconsciously project own mental models.

F) Personas also work as reality-checks, helping designers keeping the focus on the limited subset of users that have been deemed "primary".

G) Proposed designs, features and solutions can be evaluated against the needs of individual persona models.

Personas have also been criticized mostly because if they are fictional, they have no clear relationship to real customer data and therefore any data gathered cannot be considered scientific [1]. In any case, in order to apply this design method to computer games some changes are necessary.

\section{From Persona to Play-Persona}

Play-personas are further defined as clusters of preferential interaction (what) and navigation (where) attitudes, temporally expressed (when), that coalesce around different kinds of inscribed affordances in the artefacts provided by game designers. This means that personas can no longer just be limited to narrative descriptions of motivations, needs and desires distilled in ethnographic interviews.

The persona hypotheses emerge as a relation of parameters from the set of interaction and navigation possibilities that the game-rules and game spaces can afford [22]. Personas can be augmented and strengthened by a quantifiable, parametric, datadriven perspective. Furthermore, if directly coupled with instrumentation data in the form of gameplay metrics gathered from game engine software during play sessions, play-personas can provide a powerful evaluation tool to confirm whether a certain hypothesis also turns out to represent a sizeable slice of players. That is why playpersonas are both theoretical models of ideal users (metaphor) and data-driven representations of player behaviours (lens). 


\subsection{Gameplay Metrics}

Gameplay metrics are instrumentation data extracted from computer game engines during play. The collection of quantitative data about user-product interaction is an established method within Human-Computer Interaction [24, 25], but has only recently been adapted to user testing in computer game development [10,22]

The type of game instrumentation data relevant to persona modeling is gameplay metrics, which are measures of game-player interaction. Gameplay metrics can be recorded for any type of user-initiated behavior where interaction takes place in or with the virtual environment; as well as behaviors initiated by agents and systems operating in the virtual environment but which are not controlled by the player, e.g. autonomous agents. Tracked and logged actions can vary from lowlevel data such as button presses to in-game behavior. For example, tracking the location of a player through the virtual environment, the use of specific character skills, weapons, hit probability etc. Gameplay metrics analysis provide the ability to generate highly detailed, objective and quantitative analyses about player (end user) behavior.

Metrics analysis can be used to evaluate user behavior, and the data type forms a supplement to existing methods for user-oriented research and -testing in the game industry, such as usability testing, playtesting, focus groups and similar qualitative/semi-quantitative approaches. Different methods have different strengths and weaknesses. For example, usability testing focuses on measuring the ease of operation of a game. Playability testing explores if users have a good playing experience. Gameplay metrics analysis offers however detailed insights into how the users are actually playing the games being tested. Furthermore, gameplay metrics can be used to develop data-driven persona models.

\subsection{Play-Persona as Metaphor}

A metaphor is a rhetorical device that allows describing something unknown by transferring attributes from a known entity. Metaphors are utilized before the accumulation of experience, in a similar way personas allow designers to "imply" unknown player behaviour in the process of creating digital games, i.e. by pre-defining the ideal playpatterns possible in the game in question and design to accommodate these. It is the case in Tomb Raider Underworld [18], where players can choose between different identically optimal strategies to progress and express preferences for some modes of interaction and navigation instead of others. Play-personas as design tools represent an expectance of how players would like to craft their experience. As metaphors, play-personas are hypotheses that emerge as relations of parameters from the set of possibilities that the game can afford.

Designers can use personas as categories of behaviours prior to a playable version of the game in order to plan coherent navigation and interaction modes. They are also precious as guides to select which variables are interesting enough to monitor as game metrics, which will lead to the creation of play-personas as lens. 


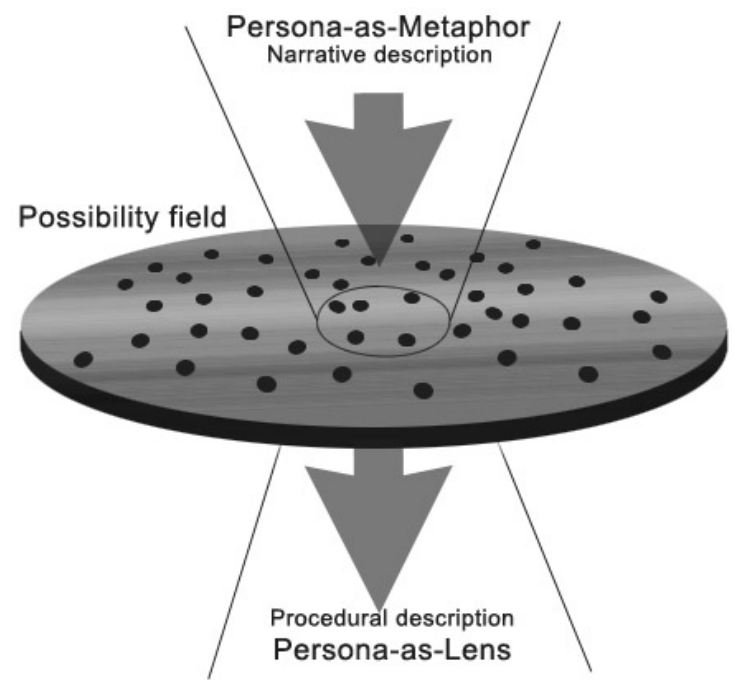

Fig. 1. Two sides of the same coin: persona as metaphor and lens. The black dots on the "possibility-field" plane represent game mechanics; thanks to the a-priori description of the personaas-metaphor a certain subset is individuated. This persona hypothesis can be checked against metric data gathered from players and inform the creation of persona-as-lens.

\subsection{Play-Persona as Lens}

Lens is here intended as the choice of a context (Ümwelt) from which to sense, categorize, measure or codify experience. Lenses are utilized to examine the accumulated experience. Gameplay metrics data can form the basis of defining data-driven personas during game testing. As lenses, play-personas are derived from game metrics gathered from players, after they have been interpreted as clusters of similar behaviours. Personas can be used as tools when evaluating games by comparing the goals set by the designers with those of the players. By comparing designers' and players' goals it is possible to check whether the game design actually supports and facilitates the planned experience in practice, and if any new personas emerge from the userinteraction with the game software. Analyzing game metrical data with multivariate statistical tools can provide a way of discovering patterns in the usage of game elements and features, thus enabling the building of personas of how players interact with the game, and whether the game design facilitates the specific play patterns of the personas assumed as hypotheses. It's a sense-making perspective, a code that allows extracting meaning form an otherwise unclear list of numbers.

Playstyles (or patterns of play) are possible ways in which certain subsets of the rules and mechanics provided by the game can be combined. Player that maintains consistent choices of styles eventually identify with a play-persona.

Personas are aggregate description of possible player behaviour both in theory, as an expectation of the designer - "a priori" metaphor - and in practice, as a description of what actual, real players do during a play session - "a posteriori” lens (Fig. 1). 


\section{Tomb Raider Underworld: A Case Study}

Taking as a concrete example the game Tomb Raider Underworld [5a] it becomes easier to see the two sides of the play-persona. The spaces, rules and mechanics of the game indicate few important skills necessary to identify personas:

- Shooting: Indicated by high or low number of deaths inflicted to enemies \& animals (interaction with NPC)

- Jumping: Indicated by high or low number of deaths caused by falling, drowning, being crushed (navigation)

- Puzzle solving: Indicated by high or low requests for help to solve puzzles (interaction with the world)

Player can have "high" or "low" skills for each of the parameters individuated. Keeping these values down to two, although loosing granularity and resolution, simplifies immensely the possibility space.

\begin{tabular}{|c|c|c|c|c|c|c|c|c|}
\hline & Expert & Rookie & Grunt & Athlete & Chess-player & \multicolumn{3}{|c|}{ Hybrid personas } \\
\hline Shooting & + & - & + & - & - & + & - & + \\
\hline Jumping & + & - & - & + & - & + & + & - \\
\hline Puzzles & + & - & - & - & + & - & + & + \\
\hline
\end{tabular}

Fig. 2. Table showing how the three parameters unfolded in the two values (+ and -) map the possibility space of the game Tomb Raider Underworld

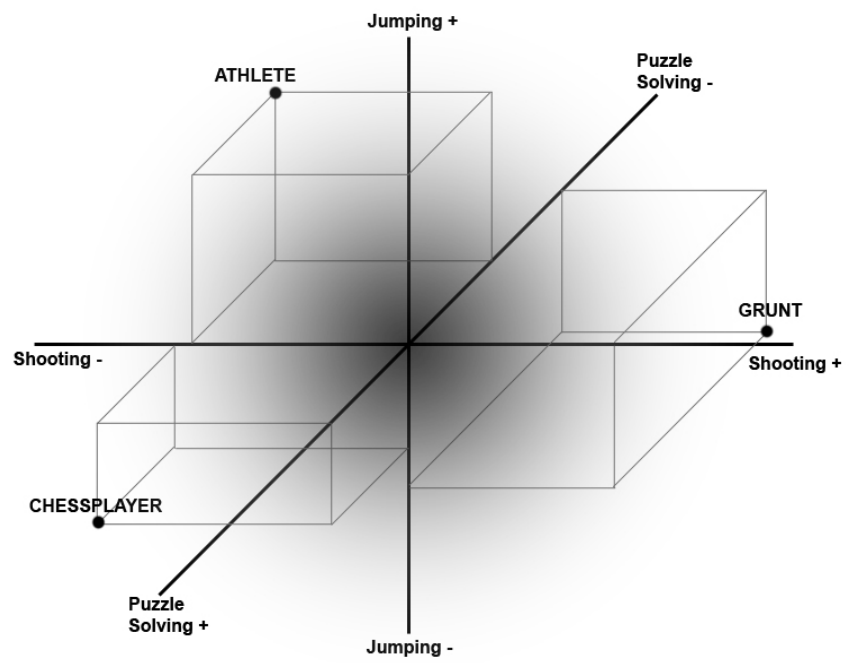

Fig. 3. Possibility space and the three play-personas: Grunt, Athlete and Chess-player. The axes can be defined abstractly such as "+" and "-" prior to a playable version of the game, when personas are used to imply user behavior patterns in the design. However, the axes can also be defined based directly on collected gameplay metrics. For example, the percentage of total puzzles solved. This provides a means for defining detailed quantitative components of the persona models, and even post-launch data-driven analysis of whether these personas emerge in the player behavior. 
Mapping the possibility space consists of exploding all the combinations of parameters underlying the skills listed before: shooting, jumping and puzzle solving.

Each of the parameters individuates an axe of an n-dimensional space, where $n$ equals the total number of variables worked with in the given situation. In this specific case there are only two possible values that each parameter can assume: plus or minus. Hence the total amount of combinations is the number of values that each parameter can assume, at the power of the number of all the parameters found: Two at the power of three for a total amount of eight combinations. This initial unfolding of gameplay parameters maps the a-priori possibility space within which personas will be found (Fig. 2).

At this point it is possible to select which personas-as-metaphors will guide the design process. Play-personas have been defined as extreme cases, one-sided accentuations that delimit the field, statistically most of the players will be identified by one of the hybrid personas, but these exaggerations are helpful both during design, to frame the kind of experience targeted by the game, and during post production, to evaluate how players actually relate to the game. Exaggerating one-sidedly the three parameters individuated lead to three personas: The Athlete, the Grunt and the Chess-player (Fig. 3).

\subsection{Grunt Persona}

Narrative description: this persona obviously excels at shooting. Grunts particularly enjoy fights and are quite good at them, they are interested in twitch stimulation, taking pride on physical domination, at the same time they might be not so precise with movements, hence the risk of failing at jumps. They might find slightly annoying tight jumping puzzles. Grunts are not interested in spending time figuring out solutions to puzzles, they are more prone to bypass cerebral strain if possible.

Procedural description:

Shooting: +

Jumping: -

Problem solving: -

\subsection{Athlete Persona}

Narrative description: athletes enjoy exploration of the environment; they will try to avoid fights if at all possible. Considering the amount of skill involved in performing precise jumps we can assume that they are players with experience and are comfortable with navigation controls, this points towards a high proficiency. Taking for granted proficient navigation skills, it is possible to assume a good sense of direction, athletes will rarely loose direction and will display relatively fast completion times, this also reflects on relatively few requests for help for spatial puzzles

Procedural description:

Shooting: -

Jumping: +

Problem solving: -

\subsection{Chess-Player Persona}

Narrative description: Chess-players get their biggest kicks out of solving puzzles, they will log almost no requests for help, there will be very few deaths caused by 
mistakes in solving puzzles, they also possesses a good sense of direction that helps a lot in navigating the environments leading to few deaths caused by the environment. At the same time they might not necessarily be good at precise jumps hence they could die several times by falling. They are not expected to show particular skills with a gun. They could resort to asking for hints to solve certain puzzles, but never to complete answers.

Procedural description:

Shooting: -

Jumping: -

Problem solving: +

\section{Play-Personas as Relations between Game Parameters}

Now that all the possible combinations have been manifested it is possible to plot them as relations between parameters (Fig. 4).

The personas as metaphors, defined as one-sided accentuations, form the hypotheses around which game and level designers plan, produce and test their work. Because of the historical legacy of the Tomb Raider franchise, it could be expected that there will be a bias of players expecting physical and mental challenges in the form of jumping and puzzle solving experiences. Even though the shooting component has always been a part of the Tomb Raider series of games, it has never been the main focus, and players looking for a full fledged shooting experience would probably pick a different title, nevertheless the developers consciously decided to strengthen the combat elements and include a varied array of enemy NPCs for players to express shooting tendencies.

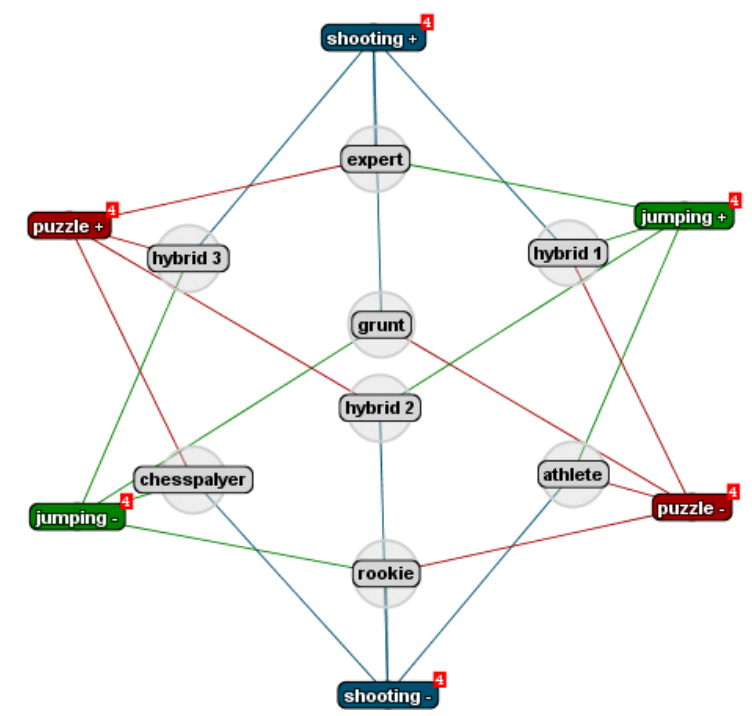

Fig. 4. Persona hypotheses emerge as relations between parameters that have been derived from gameplay mechanics 


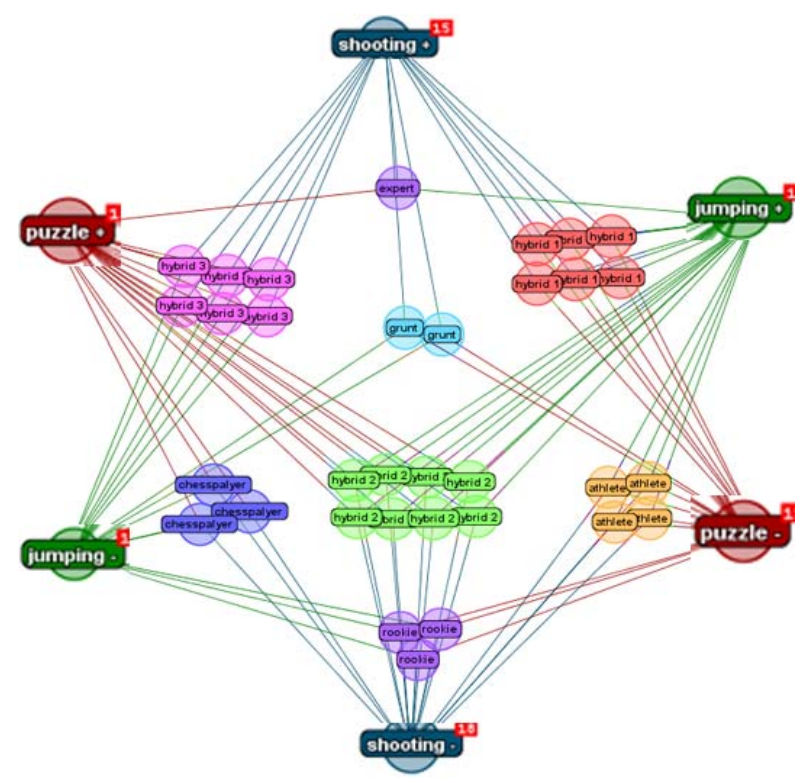

Fig. 5. Personas as lenses show the statistical layering of the population of players condensed into clusters of similar behavior profiles

The next step consists of individuating which game mechanics and variables translate as high or low puzzle solving, shooting and jumping skills, and how these can be tracked via gameplay metrics.

For example, players have the possibility to request hints and clues on how to solve puzzles in the game or they can ask directly for the complete answer via the Help On Demand-system. Thanks to the metrics tracking system developed internally at EIDOS [22], it is possible to collect data regarding players' behaviour. The decisions of hundreds of thousands players around the world to ask for help solving puzzles can therefore be collected, analyzed and form the basis for detailed persona models that relate directly to game mechanics. This information helps designers answering straight forward questions such as "where do players get stuck more often?", however, gameplay metrics datasets - depending on the variables tracked - also provide statistical evidence to the subdivision of players in the various persona-defined categories (Fig. 5).

For Tomb Raider: Underworld, data on player progression, level completion times, locations of player death, requests through the Help-on-Demand system, causes of death and a variety of other variables are tracked. Via the application of multivariate statistical methods such as cluster analysis, factor analysis and population statistical methods such as ordination/correspondence analysis, patterns of player behavior in the data can be sought out. Additionally, neural networks, decision three analysis and similar techniques can be used to locate data-driven patterns. This permits game developers to check how and if the pre-defined persona models (as metaphor) actually emerge in the way the end users are playing the game, i.e. as data-driven personas-aslens [22]. 


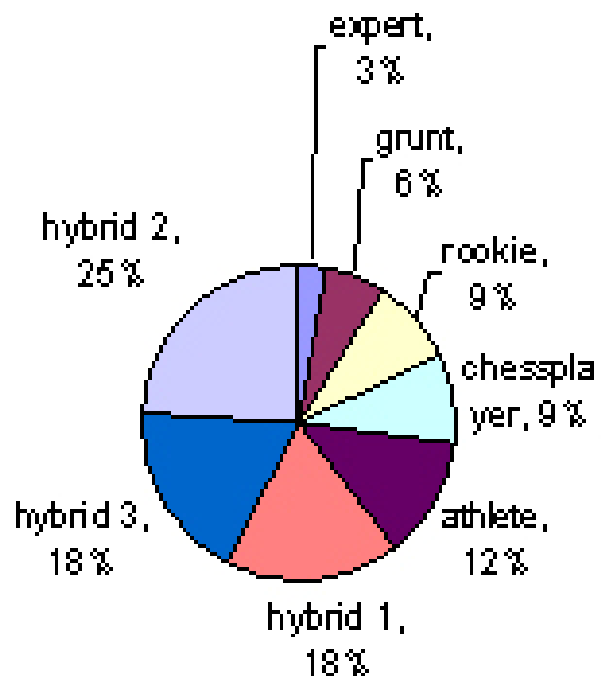

Fig. 6. Distribution of players according Tomb Raider Underworld persona profiles

In the case example for Tomb Raider: Underworld, it is apparent that the large majority of players do not fit under the umbrella of the three personas used as hypotheses, but rather is to be found in the profiles of usage defined by the hybrid personas (Fig. 6).

It is in this phase that persona hypotheses are checked against the numbers: As expected the Grunt is not very well represented, while the Athlete is the largest "pure" persona. This kind of information generates knowledge that has both short and long term effects: On a concrete level, the information provides valuable feedback to level designers, who can use the input to modify a level to achieve better balance and facilitate the different persona models; to obtaining a greater insight on the landscape of possible player types and eventually make games that can cater for a broader audience.

\section{General Applicability of the Method in Extreme Cases}

The suggested framework could potentially come short of being in any measure exhaustive when applied either to puzzle games with one solution or extremely linear games with very few areas designed for the player to make non-trivial choices. For example, in games such as Braid [6a], where there is only one way to solve the puzzles, it makes no sense to even talk about play-styles let alone personas: the player's sole duty is to second guess the designer's mind and therefore push forward the story. Similarly, in classic point-and-click adventure games, players had to advance the story by collecting and putting together a narrative that the designers exploded and spread around the game world, with no influence on what to do nor how to do it. 
Even modern AAA-level games sometimes fail to provide non trivial choices, collapsing all the potential personas into only one profile that players have to conform to in order to proceed. This type of games would not particularly benefit from the application of the method, but it's important to notice how they do not represent the majority of titles on the market, often populating the casual end.

Beside these considerations, the play-persona framework is directly useful in situations where players have more than one way to accomplish a task, any time that the combination of different game-rules in different ways could give rise to something similar to "play style", every time that players are allowed to express a preference towards a course of action instead of another, even if this only means choosing between killing a NPC with a silenced gun or with poison, as is the case in the Hitman series developed by IO Interactive.

\section{Conclusions}

A key design challenge in an increasing number of game development projects is to ensure optimal experience for a variety of player preferences, in order to reach as broad a target audience as possible $[11,15]$. In the above, a tool for addressing this requirement has been presented in the form of play-persona frameworks. These function both as models of preliminary hypothesis of in-game behaviour, and a means for categorizing and analyzing character-bound gameplay metrics variables. Playpersonas have been shown to be a tool for informing the process of developing and testing a diversity of play behaviours in a computer game.

The play-persona framework aggregates data in a way that binds ludic and narrative aspects of the game. In this paper a case study has been presented that shows how play-personas allow designers to expand their role from mere dealers of rewards and punishments, disseminating challenges and skills for a general undistinguished audience, to become crafters, orchestrators of experiences and weavers of playing modes.

Acknowledgements. The authors would like to extend their gratitude to colleagues at IO Interactive, Crystal Dynamics, the Danish Design School and the IT University of Copenhagen.

\section{References}

1. Chapman, C.N., Milham, R.: The personas' new clothes: methodological and practical arguments against a popular method. In: Proceedings of Human Factors and Ergonomics Society 50th Annual Meeting, pp. 634-636 (2006)

2. Cooper, A.: The Inmates Are Running the Asylum. SAMS Publishing, Indianapolis (2004)

3. Cooper, A., Reimann, R., Cronin, D.: About Face 3: The Essentials of Interaction Design. Wiley Publishing, Indianapolis (2007)

4. Damasio, A.: Descartes' Error: Emotion, Reason, and the Human Brain. Penguin, London (2005) 
5. Davis, J., Steury, K., Pagulayan, R.: A survey method for assessing perceptions of a game: The consumer playtest in game design. Game Studies: The International Journal of Computer Game Research 5 (2005)

6. Eco, U.: The Role of The Reader. Indiana university Press, Bloomington (1984)

7. Frijda, N.H.: The Laws of Emotion. Lawrence Erlbaum Associates Publishers, Mahwah (2007)

8. Goffman, E.: The Presentation of Self in Everyday Life. Penguin, London (1990)

9. Iser, W.: The Implied Reader. Johns Hopkins Paperback Editions, London (1978)

10. Kim, J.H., Gunn, D.V., Schuh, E., Phillips, B.C., Pagulayan, R.J., Wixon, D.: Tracking Real-Time User Experience (TRUE): A comprehensive instrumentation solution for complex systems. In: Proceedings of CHI, pp. 443-451 (2008)

11. Lazzaro, N.: Why We Play Games: Four Keys to More Emotion Without Story (2004), http://www. xeodesign.com/xeodesign_whyweplaygames.pdf

12. Mandryk, R.L., Inkpen, K.M., Calvert, T.W.: Using Psychophysiological Techniques to Measure User Experience with Entertainment Technologies. Behaviour \& Information Technology 25(2), 141-158 (2006)

13. Medlock, M.C., Wixon, D., Terrano, M., Romero, R.L., Fulton, B.: Using the RITE method to improve products: A definition and a case study. In: Proceedings of UPA (2002)

14. Nacke, L., Lindley, C.A.: Flow and Immersion in First-Person Shooters: Measuring the player's gameplay experience. In: Proceedings of the 2008 Conference on Future Play: Research, Play, Share, pp. 81-88 (2008)

15. Pagulayan, R.J., Keeker, K., Wixon, D., Romero, R.L., Fuller, T.: User-centered design in games. In: The human-computer interaction handbook: fundamentals, evolving technologies and emerging applications, pp. 883-906. Lawrence Erlbaum Associates, Philadelphia (2003)

16. Pine II, J.: Mass Customization: The New Frontier in Business Competition. Harvard Business Press (1992)

17. Ravaja, N., Saari, T., Laarni, J., Kallinen, K., Salminen, M., Holopainen, J., Järvinen, A.: The Psychophysiology of Video Gaming: Phasic Emotional Responses to Game Events. In: DiGRA conference Changing views: Worlds in play. Digital Games Research Association Press (2005)

18. Ravaja, N., Saari, T., Turpeinen, M., Laarni, J., Salminen, M., Kivikangas, M.: Spatial presence and emotions during video game playing: Does it matter with whom you play? Presence: Teleoperaters and Virtual Environments 15(4), 381-392 (2005)

19. Sanders, E.B.-N.: Scaffolds For Building Everyday Creativity, in Design for Effective Communications: Creating Contexts. Allworth Press, New York (2006)

20. Shils, E.A., Finch, E.A.: The methodology of the social sciences. Free Press, Glencoe (1997)

21. Swain, C.: Master Metrics: The Science Behind the Art of Game Design. Presentation at NLGD Conference, Utrecht, Holland (2008)

22. Tychsen, A., Canossa, A.: Defining Personas in Games Using Metrics. In: Proceedings of Future Play 2008, pp. 73-80 (2008)

23. Ó, Reilly, T.: What Is Web 2.0 - Design Patterns and Business Models for the Next Generation of Software, September 30 (2005), http: / / www . oreillynet. com/pub/a/ oreilly/tim/news/2005/09/30/what-is-web-20.html

24. Goetz, P.: Too Many Clicks! Unit-Based Interfaces Considered Harmful. Gamasutra, August 23 (2006)

25. Hilbert, D.M., Redish, J.C.: A practical guide to usability testing. Intellect books, Bristol (1999) 


\section{Ludology}

1a. Left4Dead, Valve, Electronic Arts, (2008)

2a. Little Big Planet, Media Molecule, Sony (2008)

3a. Spore, Maxis, Electronic Arts (2008)

4a. SingStar, SCE London, Sony (2008)

5a. Tomb Raider Underworld, Crystal Dynamics, EIDOS, (2008)

6a. Braid, Number None Inc. (2008) 